\title{
Guest editors introduction to the special issue for the ECML PKDD 2018 journal track
}

\author{
Jesse Davis ${ }^{1}$ D B Bjørn Bringmann ${ }^{2} \cdot$ Elisa Fromont $^{3} \cdot$ Derek Greene $^{4}$
}

Received: 29 June 2018 / Accepted: 6 July 2018 / Published online: 20 July 2018

(c) The Author(s) 2018

This special issue contains papers accepted for the Machine Learning Journal as part of the ECML PKDD 2018 journal track. The journal track began at ECML PKDD 2013 with the goal of offering authors the opportunity to have a journal publication while still being able to present their work during a conference talk. Through the track, authors have the opportunity to submit a paper to either the Machine Learning Journal or the Data Mining and Knowledge Discovery journal that, if accepted, would appear in a special issue of the respective journal. Authors of accepted journal track articles present their work at the ECML PKDD 2018 conference which takes place in Dublin, Ireland from September 10 through 14, 2018.

The goal of the journal track is to solicit high-quality, novel, and mature work that meets the standard of a journal paper while still being amenable to a conference presentation. As such, survey papers and extensions of previously published conference papers were not eligible for the journal track. The ECML PKDD 2018 journal track was organized around five submission deadlines that were roughly spaced five weeks apart. The first submission deadline was on August 27, 2017 and the last one was on January 28, 2018.

In total, 64 papers were submitted to the Machine Learning Journal of which 13 have been accepted in time to be included in this special issue. Additionally, the special issue contains four papers that were submitted to the journal track for ECML PKDD 2017, but were still under going the review process when that special issue was finalized. These papers were edited by Kurt Driessens, Dragi Kocev, Marko Robnik-Šikonja, and Myra Spiliopoulou. The accepted papers cover a multitude of topics within machine learning such as learning

Jesse Davis

jesse.davis@cs.kuleuven.be

Bjørn Bringmann

bjoern_bringmann@mckinsey.com

Elisa Fromont

elisa.fromont@irisa.fr

Derek Greene

derek.greene@ucd.ie

1 Department of Computer Science, KU Leuven, 3001 Leuven, Belgium

2 McKinsey \& Company, Inc., Munich, Germany

3 University Rennes, Inria, CNRS, IRISA, 35000 Rennes, France

4 University College Dublin, Dublin, Ireland 
in probabilistic models, reinforcement learning, automated machine learning, decision tree learning, deep learning, optimization, clustering, and preference learning among others.

Many individuals contributed to the success of the journal track. First, we would like to thank all of the authors who submitted papers. Second, we would like to thank the members of our Guest Editorial Board ${ }^{1}$ and other reviewers who provided timely and high-quality reviews. Third, we acknowledge the help and gracious support of Peter Flach (Editor-inChief of the Machine Learning Journal), Johannes Fürnkranz (Editor-in-Chief of the Data Mining and Knowledge Discovery journal), and Melissa Fearon (the Senior Editor of Springer responsible for these journals). Finally, we would like to extend our great appreciation to this year's general chairs and last year's journal track chairs for providing valuable guidance, support, and advice throughout the process. We hope that the readers enjoy the papers in this issue!

Publisher's Note Springer Nature remains neutral with regard to jurisdictional claims in published maps and institutional affiliations.

${ }^{1}$ http://www.ecmlpkdd2018.org/guesteditorial/. 